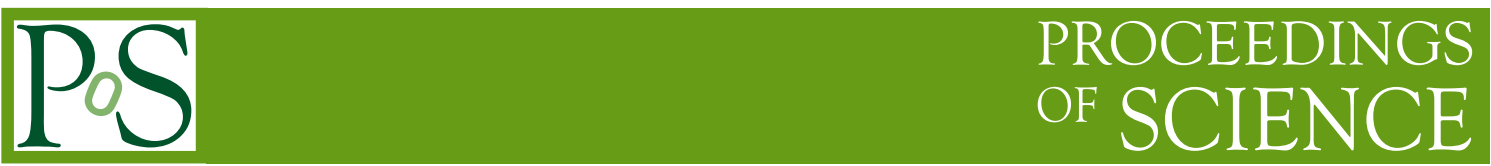

\title{
HERAFitter - an open source QCD fit framework
}

\author{
Ringailè PLAČAKYTÉ* \\ on behalf of the HERAF itter team \\ DESY (Deutsches Elektronen-Synchrotron) \\ E-mail: ringaile@mail.desy.de
}

\begin{abstract}
The patron distribution functions (PDFs) of the proton due to their non-perturbative nature are determined empirically by fitting experimental observables. The HERAFitter project aims at providing a framework for QCD analyses related to proton PDFs. The framework includes various modules and interfaces enabling a large number of theoretical and methodological options. It also alows to study the impact of the new experimental data on the PDFs from $e p, p p$ and $p \bar{p}$ processes. The fast development of the project involves active communication with theorists and close collaboration with the main PDF fitting groups. Full information about the project, downloads and documentation can be found in http://herafitter.org.
\end{abstract}

XXI International Workshop on Deep-Inelastic Scattering and Related Subjects 22-26 April, 2013

Marseilles, France

${ }^{*}$ Speaker. 


\section{HERAFitter project}

According to the factorisation theorem, the hard-scattering cross section is a convolution product of the parton distribution functions (PDFs) and the perturbatively calculable hard scattering coefficients. PDFs, which cannot be predicted by the theory of Quantum Chromodimanics (QCD), are generally determined by a fit to data from experimental observables in various processes, using the DGLAP evolution equation [1].

HERAFitter project is an open source QCD fit framework designed for the extraction of parton distribution functions of the proton. The framework includes large number of theoretical models and allows to study the impact of the new experimental data on the PDFs. A schematic structure of the HERAFitter code is illustrated in figure 1 and explained in the following text. Further information about the project with available releases and documentation (manual and release notes) can be found in http://herafitter.org.

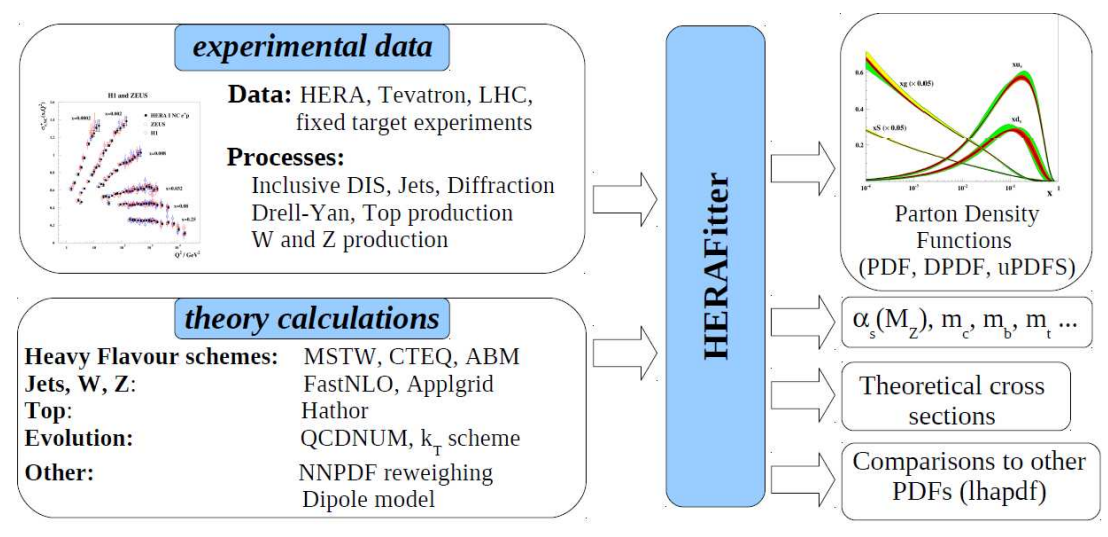

Figure 1: Schematic structure of the HERAFitter program: The "experimental data" block illustrates variety of processes which can be included into the framework and are matched to the corresponding theoretical calculation illustrated in the block below; after the QCD fit is performed the resulting PDFs together with theoretical cross sections are provided as shown on the right side of the figure.

\subsection{Functionality}

The procedure to determine PDFs in the HERAFitter framework is following: First, the PDFs are parametrised at the starting scale $Q_{0}^{2}$ chosen to be below the charm mass threshold, then fits to the experimental data are performed evolving parametrised PDFs using DGLAP evolution in the $\overline{M S}$ scheme as implemented in the QCDNUM [2] program. The evolution is available at leading (LO), next-to-leading (NLO) or next-to-next-to-leading (NNLO) order.

During the fitting procedure, the measured and predicted cross sections together with their corresponding errors are used to build a $\chi^{2}$ and determine the PDF parameters. The main functionality of the code which includes the treatment of the experimental data, calculation of the theory predictions, $\chi^{2}$ minimisation, parametrisation and optional tools are summarised below.

All main experimental data sets relevant for PDFs can be fitted within the HERAFitter framework: Inclusive cross sections from HERA DIS and fixed target experiments, Drell-Yan, jet production data (ep, $p p$ and $p \bar{p})$ and heavy quark structure functions. The DIS structure functions 
may be computed in a variety of heavy quark schemes including the fixed-flavour (FFN) and variable flavour number (VFN) schemes. VFN schemes with various treatments for the heavy quark thresholds include the Thorne Roberts (TR) scheme at LO, NLO and NNLO [3, 4] as provided by the MSTW group, the ACOT scheme at LO and NLO as provided by the CTEQ group. The QCDNUM also provides the calculations of the DIS structure functions in the zero-mass the FFN schemes. The FFN scheme is alternatively available and via the OPENQCDRAD [5] interface in which the running mass definition [6] is implemented.

For $p p$ Drell Yan processes one of two available implementations can be chosen in HERAF itter. The first implementation uses calculations at LO which can be extended to NLO using k-factors. Alternatively, one can obtain the NLO predictions directly by using APPLGRID [8] technique which rely on the factorisation theorem by decoupling the hard scattering coefficients from PDFs allowing to store them on the grid. An independent treatment for the electro-weak corrections is applied as k-factors, using external packages such as SANC and FEWZ to calculate them. APPLGRID (or FastNLO [7]) technique can also be used as fast input to NLO jet cross sections.

The program HATHOR [9] interfaced to HERAFitter provides the calculation of the total $t \bar{t}$ cross section at $p \bar{p}$ and $p p$ colliders up to approximate NNLO accuracy.

Various forms of $\chi^{2}$ can be chosen in the HERAFitter for the minimisaton procedure which are based on the use of nuisance parameters or on the full covariance matrix. For a single data set, the $\chi^{2}$ function is defined in a simple form

$$
\chi^{2}=\sum_{i} \frac{\left[m^{i}-\sum_{j} \gamma_{j}^{i} m^{i} b_{j}-\mu^{i}\right]^{2}}{\left(\delta_{i, \mathrm{stat}} m^{i}\right)^{2}+\left(\delta_{i, \mathrm{uncor}} m^{i}\right)^{2}}+\sum_{j} b_{j}^{2}
$$

or can be evolved in the so called "scaled" form:

$$
\chi^{2}=\sum_{i} \frac{\left[m^{i}-\sum_{j} \gamma_{j}^{i} m^{i} b_{j}-\mu^{i}\right]^{2}}{\delta_{i, \mathrm{stat}}^{2} \mu^{i}\left(m^{i}-\sum_{j} \gamma_{j}^{i} m^{i} b_{j}\right)+\left(\delta_{i, \text { uncor }} m^{i}\right)^{2}}+\sum_{j} b_{j}^{2}
$$

Here $\mu^{i}$ is the measured central value at a point $i$ with relative statistical $\delta_{i, s t a t}$ and relative uncorrelated systematic uncertainty $\delta_{i, u n c}$, the quantity $m^{i}$ is the theoretical prediction, $\gamma_{j}^{i}$ represents relative correlated systematic uncertainties and $b_{j}$ their shifts. In the case of the covariance matrix, the $\chi^{2}$ function takes the form

$$
\chi^{2}=\sum_{i j}\left(m^{i}-\mu^{i}\right) C_{\mathrm{tot} i j}^{-1}\left(m^{j}-\mu^{j}\right)
$$

where the $C_{\mathrm{tot} i j}^{-1}$ is the total covariance matrix given by the sum of the statistical and systematic covariance matrices. In addition, the different approaches can be combined together, e.g. some systematic uncertainties can be treated using the matrix method, others can be treated using nuisance parameters.

Several different parametrisation forms for the PDFs at the staring scale can be chosen in the HERAFitter. First, the standard functional form can be used to parametrise PDFs, second, the bi-log normal functional form is available and third, forms based on generalised polynomials such 
as the Chebyshev can be used.

Alternative way to study the impact of the experimental data on PDFs without performing a full QCD fit is to use Bayesian reweighting. Bayesian reweighting can be used with Monte Carlo method as first employed by the NNPDF Collaboration [10] or using the Hessian Eigenvector Method as proposed in [11].

The HERAF itter provides PDFs in a standard LHAPDF format which can be used by theoretical calculation and Monte Carlo simulation programs. Basic plotting tools and several examples of the fits are included in the HERAFitter package.

\subsection{Latest developments (HERAFitter-0.3.1)}

The latest developments which are included in the newest HERAF itter release (HERAFitter0.3.1 beta) and presented in the DIS2013 conference are summarised below:

- improved treatment of the experimental data uncertainties which can be treated as asymmetric (using Toy MC method), as Offset method (the minimisation is performed without taking into account correlated uncertainty sources but nuisance parameters are shifted by \pm 1 in the uncertainty determination) and using the covariance matrix representation for data sets which have uncertainties reported in this form;

- to study the possible bias introduced by the parametrisation form, a flexible PDF parametrisation and MC method now can be employed. There are two regularisation techniques, data driven and external regularisation based on the $\chi^{2}$ penalty term, which can be used to estimate parametrisation induced biases;

- PDF Bayesian reweighting based on eigenvectors (Hessian method) as explained in the section above,

- unintegrated PDFs based on the kT factorisation (CCFM) evolution as an alternative approach to collinear DGLAP evolution;

- a Bartels-Golec-Kowalski(BGK) [12] dipole model which takes into account the effects of DGLAP evolution;

- inclusion of additional data sets from LHC and Tevatron and other updates like upgraded interface format to FastNLO and newer electro-weak DIS program.

\subsection{Results obtained using HERAF itter}

The HERAFitter framework is actively used by HERA and LHC experiments. At HERA, the results of QCD analyses using HERAF itter are published in the recent combination of charm production measurements in DIS [13] and inclusive $\mathrm{H} 1$ measurements at high $Q^{2}$ with longitudinally polarised lepton beams [14]. The HERAFitter framework has been used in the QCD studies with $Z$ and $W$ cross sections measured in ATLAS to determine the strange quark density of the proton [15]. Another QCD analysis was performed on the inclusive ATLAS jet data at $\sqrt{s}=2.76$ $\mathrm{TeV}$ compared to $\sqrt{s}=7 \mathrm{TeV}$ [16]. At CMS, there are several analyses which use HERAFitter for PDF constraints, i.e. inclusive jets, DY and W+charm data. From the theory side, the work is ongoing on updating the ACOT scheme module (in collaboration with CTEQ group members), 
inclusion of photon PDF in QCDNUM (on former a publication is foreseen). The QCD studies of LHeC data have been also performed with HERAFitter and published in [17].

\subsection{Summary}

HERAFitter contains all necessary ingredients to study the proton PDFs, it incorporates variety of different data processes and theory calculations, contains many useful tools and is an optimal platform for various benchmarking studies. HERAFitter is the first open source package to perform PDF fits and is actively used by experimental and theoretical high energy physics communities.

\section{References}

[1] V.N. Gribov, L.N. Lipatov, Sov. J. Nucl. Phys. 15, 438, 675 (1972),

G. Altarelli, G. Parisi, Nucl. Phys. B 126,298, (1977),

G. Curci, W. Furmanski, and R. Petronzio, Nucl. Phys. B 175, 27 (1980),

W. Furmanski and R. Petronzio, Nucl. Phys. B 97, 473 (1980),

S. Moch, J. Vermaseren, and A. Vogt, Nucl. Phys. B 688, 101 (2004),

A. Vogt, S. Moch, and J. Vermaseren, Nucl. Phys. B 691, 129 (2004).

[2] M. Botje (2010), http://www.nikef.nl/h24/qcdnum/index.html, [arXiv:1005.1481].

[3] R. S. Thorne and R. G. Roberts, Phys. Rev. D 57, 6871 (1998), [hep-ph/9709442].

[4] R. S. Thorne, Phys. Rev. D 73, 054019 (2006), [hep-ph/0601245].

[5] S. Alekhin, OPENQCDRAD, a program description and the code are available via: http://www-zeuthen.desy.de/ alekhin/OPENQCDRAD.

[6] S. Alekhin and S. Moch, Phys.Lett. B 699, 345, (2011), [arXiv:1011.5790].

[7] D. Britzger, K. Rabbertz, F. Stober, M. Wobisch, "New features in version 2 of the fastNLO project", in the proceedings of the XX International Workshop on Deep Inelastic Scattering (DIS12), 26-30th March 2012, [hep-ph/1208.3641]; http://fastnlo.hepforge.org/

[8] T. Carli et al., Eur. Phys. J C 66, 503 (2010), [arXiv:0911.2985]; https://applgrid.hepforge.org/

[9] M. Aliev et al., Comput. Phys. Commun. 182, 1034, 2011, [arXiv:1007.1327].

[10] R. D. Ball et al., Nucl. Phys. B 855, 608 (2012), [arXiv:1108.1758], R. D. Ball et al. [NNPDF Collaboration], Nucl. Phys. B 849, 112 (2011), [arXiv:1012.0836].

[11] G. Watt and R. Thorne, JHEP 1208, 052 (2012), [arXiv:1205.4024].

[12] J. Bartels, K. Golec-Biernat, and H. Kowalski, Phys. Rev. D 66, 014001 (2002), [hep-ph/0203258].

[13] H. Abramowicz et al. [H1 and ZEUS Collaborations], Eur. Phys. J C73 (2013) 2311, [arXiv:1211.1182].

[14] F. Aaron et al. [H1 Collaboration], JHEP 1209 (2012) 061, [arXiv:1206.7007].

[15] Georges Aad et al. [ATLAS Collaboration], Phys. Rev. Lett. 109 (2012) 012001, [arXiv:1203.4051].

[16] Georges Aad et al. [ATLAS Collaboration], EPJC 732509 (2013), [arXiv:1304.4739].

[17] J. L. Abelleira Fernandez et al. [LHeC Study Group], "A Large Hadron Electron Collider at CERN" J.Phys.G. 39 (2012) 075001, [arXiv:1206.2913]. 